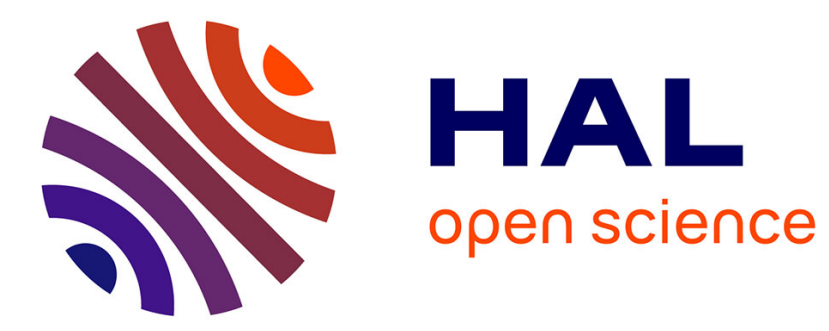

\title{
Impulsive fixed-time observer for linear time-delay systems
}

Kokou Langueh, Gang Zheng, Thierry Floquet

\section{To cite this version:}

Kokou Langueh, Gang Zheng, Thierry Floquet. Impulsive fixed-time observer for linear time-delay systems. Journal of The Franklin Institute, 2018, 355 (7), pp.3354-3366. 10.1016/j.jfranklin.2018.02.019 . hal-02057901

\section{HAL Id: hal-02057901 \\ https://hal.science/hal-02057901}

Submitted on 5 Mar 2019

HAL is a multi-disciplinary open access archive for the deposit and dissemination of scientific research documents, whether they are published or not. The documents may come from teaching and research institutions in France or abroad, or from public or private research centers.
L'archive ouverte pluridisciplinaire HAL, est destinée au dépôt et à la diffusion de documents scientifiques de niveau recherche, publiés ou non, émanant des établissements d'enseignement et de recherche français ou étrangers, des laboratoires publics ou privés. 


\title{
Impulsive fixed-time observer for linear time-delay systems
}

\author{
K. Langueh ${ }^{1}$, G. Zheng ${ }^{1}$ and T. Floquet ${ }^{1}$ \\ ${ }^{1}$ INRIA Lille-Nord Europe, 40, avenue Halley, 59650 Villeneuve d'Ascq, France
}

\begin{abstract}
This paper presents a fixed-time observer for a general class of linear time-delay systems. In contrast to many existing observers, which normally estimate system's trajectory in an asymptotic fashion, the proposed observer estimates system's state in a prescribed time. The proposed fixed-time observer is realized by updating the observer in an impulsive manner. Simulation results are also presented to illustrate the convergence behavior of the proposed fixed-time observer.
\end{abstract}

\section{Introduction}

Time-delay systems describe a wide range of dynamic processes arising often in chemical, biological and economic applications. The observer design problem of linear system without delays has already been solved in Luenberger (1966), Darouach et al. (1994), Hostetter and Meditch (1973), Hou and Müller (1992), Kudva et al. (1980), Yang and Wilde (1988) and Wang et al. (1975). However, for time-delay systems which are used sometimes to model practical applications Richard (2003), Sename and Briat (2001),Zheng et al. (2008), the observer design problem becomes not trivial when the system states and the output contain commensurate delays.

Observability and identifiability are analyzed in Bejarano and Zheng (2014) for linear time-delay systems and in Zheng et al. (2011) and Zheng et al. (2013) for nonlinear one. Concerning the observer design for time-delay systems, different techniques have been proposed in the literature, such as infinite dimensional approach Salamon (1980), polynomial approach based on the ring theory Sename (1997); Emre and Khargonekar (1982); Bejarano and Zheng (2017); Zheng and Bejarano (2017), Lyapunov function based on LMI Darouach (2001); Seuret et al. (2007) and so on. Recently, some new inequality techniques have been addressed in Chen et al. (2017a) and Chen et al. (2017b) which can be also used to design observers.

All those mentioned observers in the literature provide only asymptotic (or exponential) estimation. However, in some applications it is desired to have a faster estimation, for example in a prescribed finite time Shi et al. (2015), which is named as non-asymptotic convergence. When designing state feedback control for nonlinear systems, the non-asymptotic estimation enables us to avoid the closed loop analysis for the observer and controller, even if the separation principle is generally not satisfied for nonlinear systems. Non-asymptotic estimation has a long history, and many different approaches have been proposed in the literature, such as algebraic method 
Sira-Ramrez et al. (2014) and sliding mode technique Utkin (1992). Usually the nonasymptotic convergence, when applying sliding mode method, depends on initial conditions Zheng and Wang (2016). Some extensions have been done to provide the uniform non-asymptotic convergence with respect to the initial conditions Cruz-Zavala et al. (2011), named as 'fixed-time'. Inspired by the works of Engel and Kreisselmeier (2002); Raff and Allgower (2007) for linear systems, where the authors combined two simple Luenberger observers to construct a fixed-time observer, where the fixed time can be arbitrary assigned, this paper utilizes this idea to treat linear time-delay systems with known inputs. As an extension of Langueh et al. (2016), the fixed-time convergence of the proposed observer is achieved by updating the observers with an impulsive manner.

This paper adopts the method based on ring theory since it enables us to use some useful techniques developed for linear systems without delays Zheng et al. (2015). The following notations will be used in this paper. $\mathbb{R}$ is the field of real numbers. The set of positive integers is denoted by $\mathbb{N}$. $I_{p}$ means $p \times p$ identity matrix. $\mathbb{R}[\delta]$ is the polynomial ring over the field $\mathbb{R}$ and $\mathbb{R}^{n}[\boldsymbol{\delta}]$ is the $\mathbb{R}[\boldsymbol{\delta}]$-module whose elements are vectors of dimension $n$ and whose entries are polynomials. By $\mathbb{R}^{q \times s}[\delta]$ we denote the set of matrices of dimension $q \times s$, whose entries are in $\mathbb{R}[\delta]$. For a matrix $M(\delta)$, $\operatorname{rank}_{\mathbb{R}[\delta]} M(\delta)$ means the rank of the matrix $M(\delta)$ over $\mathbb{R}[\delta]$. The maximal order of $\delta$ in all polynomials in the matrix $M(\delta)$ is denote $\operatorname{deg}_{\delta} M(\delta)$ We denote $\operatorname{Inv}_{S}[M(\delta)]=$ $\left\{\Psi_{i}(\delta)\right\}_{1 \leq i \leq r}$ as the set of invariant factors of the Smith form of $M(\delta)$.

\section{Problem statement}

In this paper, we consider the following class of linear systems with commensurate delays:

$$
\begin{aligned}
\dot{x}(t) & =\sum_{i=0}^{k_{a}} \bar{A}_{i} x(t-i h)+\sum_{i=0}^{k_{b}} \bar{B}_{i} u(t-i h) \\
y(t) & =\sum_{i=0}^{k_{c}} \bar{C}_{i} x(t-i h)
\end{aligned}
$$

where the vector $x(t) \in \mathbb{R}^{n}$, the input vector $u(t) \in \mathbb{R}^{m}$, and the output vector $y(t) \in \mathbb{R}^{p}$, the initial function $\varphi(t)$ is a piece-wise continuous function $\varphi(t):[-k h, 0] \rightarrow \mathbb{R}^{n}(k=$ $\left.\max \left\{k_{a}, k_{b}, k_{c}\right\}\right)$ where $h$ represents the basic commensurate delay; thereby $x(t)=\varphi(t)$ on $[-k h, 0] . \bar{A}_{i}, \bar{B}_{i}$ and $\bar{C}_{i}$ are matrices of appropriate dimension.

In order to simplify the analysis, let us introduce the delay operator $\delta$ such that $x(t-k h)=\delta^{k} x(t), k \geq 0$. Let $\mathbb{R}[\delta]$ be the polynomial ring of $\delta$ over the field $\mathbb{R}$. After having introduced the delay operator $\delta$, system (1) can be written as follows:

$$
\begin{aligned}
& \dot{x}(t)=A(\delta) x(t)+B(\delta) u(t) \\
& y(t)=C(\delta) x(t)
\end{aligned}
$$

where $A(\delta)=\sum_{i=0}^{k_{a}} \bar{A}_{i} \delta^{i}, B(\delta)=\sum_{i=0}^{k_{b}} \bar{B}_{i} \delta^{i}$ and $C(\delta)=\sum_{i=0}^{k_{c}} \bar{C}_{i} \delta^{i}$.

As for $x(t, \varphi, u)$, we mean the solution of system (2) with the initial functions equal to $\varphi(t)$ and the inputs equal to $u$. In the same way, we define $y(t, \varphi, u)=C(\delta) x(t, \varphi, u)$, which is the output of system (1) when $x(t)=x(t, \varphi, u)$. 
When designing observers for time-delay system (2), we can only use $y(t)$ and its delayed values to estimate the current $x(t)$. Otherwise, the designed observer is not causal. In this sense, it is desired to use actual and past information of measurement. For this, let us firstly recall the definition of backward observability stated in Bejarano and Zheng (2014).

Definition 1. System (1) is said to be backward observable on $\left[t_{1}, t_{2}\right]$ if and only if for each $\tau \in\left[t_{1}, t_{2}\right]$, there exist $t_{1}^{\prime}<t_{2}^{\prime} \leq \tau$ such that for every initial condition $\varphi, y(t, \varphi, 0)=$ 0 for all $t \in\left[t_{1}^{\prime}, t_{2}^{\prime}\right]$ implies $x(\tau, \varphi, 0)=0$.

Concerning systems with time delays, we have also the following definition of asymptotic and non-asymptotic observers Zheng et al. (2014).

Definition 2. The dynamics

$$
\left\{\begin{array}{l}
\dot{\xi}=f(y, \xi, \delta, u) \\
\hat{x}=g(y, \xi, \delta, u)
\end{array}\right.
$$

with $y$ being the output of (2), $\xi \in \mathbb{R}^{\tilde{n}}, \hat{x} \in \mathbb{R}^{n}$ where $\tilde{n} \geq n$ and some user-chosen functions $f$ and $g$, is called as an asymptotic observer of (2) if

$$
\lim _{t \rightarrow \infty}|| \hat{x}(t)-x(t) \|=0 .
$$

It is said to be a finite-time observer if there exists $T_{s}\left(e_{0}\right)$, depending on the initial observation error $e_{0}=\hat{x}\left(t_{0}\right)-x\left(t_{0}\right)$, such that

$$
\|\hat{x}(t)-x(t)\|=0, \forall t \geq T_{s}\left(e_{0}\right) .
$$

In addition, this dynamics is named as a fixed-time observer if

$$
\|\hat{x}(t)-x(t)\|=0, \forall t \geq T_{s}
$$

where $T_{s}$ is independent of any initial observation error.

For system (2), Hou et al. (2002) proposed a simple Luenberger-like asymptotic observer and sufficient conditions were deduced to calculate the gains of the proposed observer. To the best of our knowledge, no results on fixed-time observer for system (2) have been studied in the literature. As an extension of Bejarano and Zheng (2014), the main contribution of this paper is to extend the methods proposed in Raff and Allgower (2007) and Hou et al. (2002) to treat system (2), and to deduce some sufficient conditions which enable us to present a constructive procedure to design a simple impulsive fixed-time observer.

\section{Definition and preliminary results}

Consider system (2) which is described by the polynomial matrices over $\mathbb{R}[\delta]$, let us firstly give some useful definitions of unimodularity and change of coordinates over $\mathbb{R}[\delta]$. 
Definition 3. For a given polynomial matrix $A(\delta) \in \mathbb{R}^{n \times q}[\delta]$, it is said to be left (or right) unimodular over $\mathbb{R}[\boldsymbol{\delta}]$ if there exists $A_{L}^{-1}(\delta) \in \mathbb{R}^{q \times n}[\delta]$ with $n \geq q\left(\right.$ or $A_{R}^{-1}(\delta) \in$ $\mathbb{R}^{n \times q}[\boldsymbol{\delta}]$ with $\left.n \leq q\right)$, such that $A_{L}^{-1}(\boldsymbol{\delta}) A(\boldsymbol{\delta})=I_{q}\left(\right.$ or $\left.A_{R}^{-1}(\boldsymbol{\delta}) A(\boldsymbol{\delta})=I_{n}\right)$. A square matrix $A(\delta) \in \mathbb{R}^{n \times n}[\delta]$ is said to be unimodular over $\mathbb{R}[\delta]$ if $A_{L}^{-1}(\delta)=A_{R}^{-1}(\delta)$.

Let us recall that for any polynomial matrix $D(\delta) \in \mathbb{R}^{p \times m}[\delta]$ with $\operatorname{rank}_{\mathbb{R}[\delta]} D(\delta)=k \leq$ $\min \{p, m\}$, it is known (see Hou et al. (2002)) that $D(\delta) \in \mathbb{R}^{p \times m}[\delta]$ is left unimodular over $\mathbb{R}[\delta]$ if and only if $\operatorname{rank}_{\mathbb{R}[\delta]} D(\delta)=m \leq p$ and $\operatorname{Inv}_{S}[D(\delta)] \subset \mathbb{R}$.

Definition 4. A change of coordinates $z=T(\delta) x$ with $T(\delta) \in \mathbb{R}^{n_{z} \times n}[\delta]$ is said to be bicausal if $T(\delta)$ is left unimodular over $\mathbb{R}[\boldsymbol{\delta}]$.

Then, we can define the following polynomial matrix over $\mathbb{R}[\delta]$ :

$$
\mathscr{O}_{l}(\boldsymbol{\delta})=\left[\begin{array}{c}
C(\boldsymbol{\delta}) \\
C(\boldsymbol{\delta}) A(\boldsymbol{\delta}) \\
\vdots \\
C(\boldsymbol{\delta}) A^{l-1}(\boldsymbol{\delta})
\end{array}\right] \in \mathbb{R}^{p l \times n}
$$

where $l \in \mathbb{N}$, and make the following assumption.

Assumption 1. It is supposed that there exists a least integer $l^{*} \in \mathbb{N}$ such that $\mathscr{O}_{l^{*}}(\delta)$ defined in (3) is left unimodular over $\mathbb{R}[\boldsymbol{\delta}]$, i.e., $\operatorname{rank}_{\mathbb{R}[\delta]} \mathscr{O}_{l^{*}}(\boldsymbol{\delta})=n$ and $\operatorname{Inv}_{S} \mathscr{O}_{l^{*}}(\boldsymbol{\delta}) \subset$ $\mathbb{R}$.

It is clear to see that if Assumption 1 is satisfied, then system (2) is backward observable Hou et al. (2002). In other words, the above assumption guarantees that the studied system (2) is backward observable, then we can design a fixed-time observer to estimate $x(t)$, which will be presented in the next section.

\section{Impulsive fixed-time observer}

This section proposes an impulsive fixed-time observer based on two simple coupled Luenberger-like observers. Before this, let us recall a useful result stated in Hou et al. (2002).

Lemma 1. Hou et al. (2002) There exists a bicausal change of coordinates $z=T(\boldsymbol{\delta}) x$ which transforms system (2) into the following observable normal form:

$$
\left\{\begin{aligned}
\dot{z} & =A_{0} z+F(\boldsymbol{\delta}) y+T(\boldsymbol{\delta}) B(\boldsymbol{\delta}) u \\
y & =C_{0} z
\end{aligned}\right.
$$

where the matrices $F(\boldsymbol{\delta})=\left[F_{1}^{T}(\delta), \ldots, F_{l^{*}}^{T}(\boldsymbol{\delta})\right]$, and

$$
\begin{aligned}
A_{0} & =\left[\begin{array}{ccccc}
0 & I_{p} & 0 & \cdots & 0 \\
\vdots & \vdots & \vdots & \ddots & \vdots \\
0 & 0 & 0 & \cdots & \vdots \\
0 & 0 & 0 & \cdots & 0
\end{array}\right] \in \mathbb{R}^{p l^{*} \times p l^{*}} \\
C_{0} & =\left[\begin{array}{lllll}
I_{p} & 0 & 0 & \cdots & 0
\end{array}\right] \in \mathbb{R}^{p \times p l^{*}}
\end{aligned}
$$


if and only if there exists a least integer $l^{*} \in \mathbb{N}$ such that $\mathscr{O}_{l^{*}}(\delta)$ defined in (3) is left unimodular over $\mathbb{R}[\delta]$. Moreover, the bicausal change of coordinates $z=T(\delta) x$ with $T(\boldsymbol{\delta})=\operatorname{col}\left\{T_{1}(\boldsymbol{\delta}), \ldots, T_{l^{*}}(\boldsymbol{\delta})\right\}$ is defined as follows:

$$
\left\{\begin{array}{l}
T_{1}(\boldsymbol{\delta})=C(\boldsymbol{\delta}) \\
T_{i+1}(\boldsymbol{\delta})=T_{i}(\boldsymbol{\delta}) A(\boldsymbol{\delta})-F_{i}(\boldsymbol{\delta}) C(\boldsymbol{\delta}), \text { for } 1 \leq i \leq l^{*}-1
\end{array}\right.
$$

with $F_{i}(\delta)$ being determined through the following equation:

$$
\left[F_{l^{*}}(\delta), \ldots, F_{1}(\delta)\right]=C(\delta) A^{l^{*}}(\delta)\left[\mathscr{O}_{l^{*}}(\delta)\right]_{L}^{-1} .
$$

The above lemma shows that if Assumption 1 is satisfied, then (2) can be transformed into (4) via $z=T(\delta) x$ where $T(\delta)$ is defined in (6). For the obtained observer normal form (4), consider now the following dynamics:

$$
\left\{\begin{array}{l}
\dot{\hat{z}}=L \hat{z}+P(\delta) y+T(\boldsymbol{\delta}) B(\boldsymbol{\delta}) u \\
\hat{x}=T_{L}^{-1}(\boldsymbol{\delta}) \hat{z}
\end{array}\right.
$$

where $L \in \mathbb{R}^{p l^{*} \times p l^{*}}$ and $P(\delta) \in \mathbb{R}^{p l^{*} \times p}[\delta]$ will be determined hereafter. Then the estimate error $\varepsilon_{z}=z-\hat{z}$ is governed by:

$$
\begin{aligned}
\dot{\varepsilon}_{z} & =\dot{z}-\dot{\hat{z}}=A_{0} z+F(\delta) y+T(\delta) B(\delta) u-\dot{\hat{z}} \\
& =A_{0} z+F(\boldsymbol{\delta}) C_{0} z-\left[L \hat{z}+P(\delta) C_{0} z\right] \\
& =\left[A_{0}+F(\boldsymbol{\delta}) C_{0}-P(\boldsymbol{\delta}) C_{0}\right] z-L \hat{z}
\end{aligned}
$$

Since $\left(A_{0}, C_{0}\right)$ is observable, thus there exists a constant matrix $J$ such that $\left[A_{0}-J C_{0}\right]$ is Hurwitz. By choosing $P(\boldsymbol{\delta})=J+F(\boldsymbol{\delta})$ and $L=A_{0}-J C_{0}$, then we obtain

$$
\dot{\varepsilon}_{z}=\left[A_{0}-J C_{0}\right] \varepsilon_{z}
$$

which is asymptotically convergent to zero. We would like to emphasize that this convergence is independent of delay. Note that $\varepsilon_{x}=T_{L}^{-1}(\delta) \varepsilon_{z}$, then $\varepsilon_{x}$ converges to zero as well asymptotically. According to Definition 2, the dynamics (8) is an asymptotic observer of system (2).

The following will use two simple observers of type (8) to construct an impulsive fixed-time observer for system (2). For this, let us choose two different constant matrices $J_{i}$ such that both $\left[A_{0}-J_{i} C_{0}\right]$ are Hurwitz for $i=1,2$. Then we note

$$
L_{i}=A_{0}-J_{i} C_{0}
$$

and

$$
P_{i}(\boldsymbol{\delta})=J_{i}+F(\delta)
$$

for $i=1,2$.

Define $d_{\max }=\operatorname{deg}_{\delta} P_{i}(\delta)$ for $1 \leq i \leq 2$ as the maximum degree of polynomials $P_{i}(\delta)$ with respect to $\delta$. With those matrices, for a prescribed positive constant $\sigma>$ 
$h \times d_{\max }$ where $h$ represents the basic commensurate delay in (1), we can then design the following two coupled observers:

$$
\left\{\begin{array}{l}
\dot{\hat{z}}_{i}=L_{i} \xi+P_{i}(\delta) y+T(\delta) B(\delta) u, \\
\hat{z}=\Gamma(\delta, t)\left[\begin{array}{l}
\hat{z}_{1} \\
\hat{z}_{2}
\end{array}\right] \\
\hat{z}_{1}(t)=\hat{z}_{2}(t), \forall t \in\left[-h \times d_{\max }, 0\right] \\
\hat{x}=T_{L}^{-1}(\boldsymbol{\delta}) \hat{z}
\end{array}\right.
$$

where

$$
\Gamma(\delta, t)=\left\{\begin{array}{l}
{\left[\frac{1}{2} I_{p l^{*}}, \frac{1}{2} I_{p l^{*}}\right], \text { if } t \neq \sigma} \\
{\left[I_{p l^{*}}-e^{\left(L_{2}-L_{1}\right) t}\right]^{-1}\left[-e^{\left(L_{2}-L_{1}\right) t}, I_{p l^{*}}\right], \text { if } t=\sigma}
\end{array}\right.
$$

Theorem 1. Suppose that Assumption 1 is satisfied for system (2). For a given prescribed constant $\sigma>h \times d_{\max }$, the dynamics (12) is an impulsive fixed-time observer of system (2) with the prescribed settling time $\sigma$, i.e. $\|x(t)-\hat{x}(t)\|=0$ for all $t \geq \sigma$.

Proof 1. It has been shown in the above that if Assumption 1 is satisfied for system (2), then one can always find two constant matrices $J_{1}$ and $J_{2}$ such that both $\left[A_{0}-J_{1} C_{0}\right]$ and $\left[A_{0}-J_{2} C_{0}\right]$ are Hurwitz. Note $\varepsilon_{z_{1}}=z-\hat{z}_{1}$ and $\varepsilon_{z_{2}}=z-\hat{z}_{2}$, then according to (9) we obtain

$$
\dot{\varepsilon}_{z_{1}}=\left[A_{0}-J_{1} C_{0}\right] \varepsilon_{z_{1}}=L_{1} \varepsilon_{z_{1}}
$$

and

$$
\dot{\varepsilon}_{z_{2}}=\left[A_{0}-J_{2} C_{0}\right] \varepsilon_{z_{2}}=L_{2} \varepsilon_{z_{2}}
$$

By solving (14) and (15), we get

$$
\begin{aligned}
& \varepsilon_{z_{1}}(t)=\left[z(t)-\hat{z}_{1}(t)\right]=e^{L_{1} t} \varepsilon_{z_{1}}(0) \\
& \varepsilon_{z_{1}}(t)=\left[z(t)-\hat{z}_{2}(t)\right]=e^{L_{2} t} \varepsilon_{z_{2}}(0)
\end{aligned}
$$

Due to the fact that the initial functions for $\hat{z}_{i}(t)$ for all $t \in\left[-h \times d_{\max }, 0\right]$ are the same, thus $\hat{z}_{1}(0)=\hat{z}_{2}(0)$ which implies $\varepsilon_{z_{1}}(0)=\varepsilon_{z_{2}}(0)$. Therefore, by eliminating $\varepsilon_{z_{1}}(0)$ and $\varepsilon_{z_{2}}(0)$ in $(16)$, we obtain

$$
e^{L_{2} t}\left[z(t)-\hat{z}_{1}(t)\right]=e^{L_{1} t}\left[z(t)-\hat{z}_{2}(t)\right]
$$

which gives

$$
z(t)=\left[I_{p l^{*}}-e^{\left(L_{2}-L_{1}\right) t}\right]^{-1}\left[-e^{\left(L_{2}-L_{1}\right) t}, I_{p l^{*}}\right]\left[\begin{array}{c}
\hat{z}_{1}(t) \\
\hat{z}_{2}(t)
\end{array}\right]
$$

When $t=\sigma$, the above equation is equal to

$$
z(\sigma)=\Gamma(\boldsymbol{\delta}, \boldsymbol{\sigma})\left[\begin{array}{l}
\hat{z}_{1}(\sigma) \\
\hat{z}_{2}(\sigma)
\end{array}\right] .
$$

According to the impulsive behavior defined in (14), we have

$$
\hat{z}_{1}(\sigma)=\hat{z}_{2}(\sigma)=z(\sigma)
$$


thus $\hat{x}(\sigma)=T_{L}^{-1}(\delta) z(\sigma)=x(\sigma)$, which implies that the exact value of $x(t)$ has been obtained at time $\sigma$. Finally, we always have $\|x(t)-\hat{x}(t)\|=0$ when $t \geq \sigma$.

Therefore, according to Definition 2, system (12) is an impulsive fixed-time observer for (2) with the prescribed settling time $\sigma$ independent of any initial condition.

Remark 1. Clearly, the result presented in Theorem 1 is based on the results stated in Raff and Allgower (2007) and Hou and Müller (1992). In Raff and Allgower (2007), two simple Luenberger observers were coupled together to achieve the finite-time estimation for linear time-invariant systems, while Hou and Müller (1992) proposed a Luenberger observer to achieve asymptotic estimation of linear time-delay systems. Compared to those results, Theorem 1 presented in this paper introduces a constructive way to achieve finite-time estimation for a class of time-delay systems.

If Assumption 1 for a certain $l^{*} \in \mathbb{N}$ is satisfied for system (2), then the following procedure presents how to design the proposed fixed-time observer:

Step 1: Transform system (2) into (4) and deduce $A_{0}$ and $C_{0}$ defined in (5);

Step 2: Calculate $\mathscr{O}_{l^{*}}(\delta)$ defined in (3) and its left unimodularity $\left[\mathscr{O}_{l^{*}}(\boldsymbol{\delta})\right]_{L}^{-1}$;

Step 3: Deduce $T(\delta)$ and $F(\delta)$ according to (6) and (7);

Step 4: Choose two different constant matrices $J_{1}$ and $J_{2}$ such that $L_{1}$ and $L_{2}$ defined in (10) are Hurwitz, then calculate $P_{i}(\boldsymbol{\delta})$ according to (11);

Step 5: Finally we obtain $\Gamma(\delta, t)$ according to (13) for a prescribed $\sigma \geq h \times d_{\max }$, and observer (12) can be then designed.

\section{Illustrative example}

Let us consider the following example of the form (2) with

$$
A(\delta)=\left[\begin{array}{ccc}
\delta^{2} & 1 & \delta \\
\delta & \delta & 1+\delta \\
1 & \delta & \delta^{2}
\end{array}\right], B(\delta)=\left[\begin{array}{cc}
1 & \delta \\
0 & 1 \\
1+\delta^{2} & 1
\end{array}\right]
$$

and

$$
C(\delta)=\left[\begin{array}{lll}
1 & 0 & 0 \\
\delta & 0 & 1
\end{array}\right]
$$

It can be checked that there exists $l^{*}=2$ such that Assumption 1 is satisfied. Therefore, the studied system is backward observable. In order to design the proposed impulsive fixed-time observer, we can follow the proposed procedure.

Step 1: According to Lemma 1, we obtained

$$
A_{0}=\left[\begin{array}{llll}
0 & 0 & 1 & 0 \\
0 & 0 & 0 & 1 \\
0 & 0 & 0 & 0 \\
0 & 0 & 0 & 0
\end{array}\right]
$$


and

$$
C_{0}=\left[\begin{array}{llll}
1 & 0 & 0 & 0 \\
0 & 1 & 0 & 0
\end{array}\right]
$$

Step 2: According to (3), we compute :

$$
\mathscr{O}_{l^{*}}(\boldsymbol{\delta})=\left[\begin{array}{c}
C(\boldsymbol{\delta}) \\
C(\delta) A(\boldsymbol{\delta})
\end{array}\right]=\left[\begin{array}{ccc}
1 & 0 & 0 \\
\delta & 0 & 1 \\
\delta^{2} & 1 & \delta \\
\delta^{3}+1 & 2 \delta & 2 \delta^{2}
\end{array}\right]
$$

and its left unimodular matrix over $\mathbb{R}[\delta]$ is equal to:

$$
\left[\mathscr{O}_{l^{*}}(\delta)\right]_{L}^{-1}=\left[\begin{array}{cccc}
1 & 0 & 0 & 0 \\
0 & -\delta & 1 & 0 \\
-\delta & 1 & 0 & 0
\end{array}\right] .
$$

Step 3: According to (7), we can deduce $F(\boldsymbol{\delta})$ as follows:

$$
F(\delta)=\left[\begin{array}{cc}
2 \delta^{2}+\delta & 0 \\
3 \delta^{3}+2 \delta^{2}+1 & 0 \\
-\delta^{4}-\delta^{2}+\delta & -\delta^{2}+\delta+1 \\
-2 \delta^{2}\left(\delta^{3}+\delta-1\right) & 2 \delta\left(-\delta^{2}+\delta+1\right)
\end{array}\right]
$$

which gives us, based on (6), the following $T(\delta)$ :

$$
T(\delta)=\left[\begin{array}{ccc}
1 & 0 & 0 \\
\delta & 0 & 1 \\
-\delta^{2}-\delta & 1 & \delta \\
-2 \delta^{3}-2 \delta^{2} & 2 \delta & \delta^{2}
\end{array}\right]
$$

whose left unimodular matrix over $\mathbb{R}[\boldsymbol{\delta}]$ is:

$$
T_{L}^{-1}(\delta)=\left[\begin{array}{cccc}
1 & 0 & 0 & 0 \\
2 \delta^{2}+\delta & -\delta & 1 & 0 \\
-\delta & 1 & 0 & 0
\end{array}\right]
$$

Step 4: Then, we can freely choose $J_{1}$ and $J_{2}$ such that $L_{1}$ and $L_{2}$ defined in (10) are stable. In this example, we take $J_{1}=\left[\begin{array}{cc}6 & 0 \\ 0 & 12.5 \\ 8 & 0 \\ 0 & 37.5\end{array}\right]$ and $J_{2}=\left[\begin{array}{cc}2.4 & 0 \\ 0 & 5 \\ 1.28 & 0 \\ 0 & 6\end{array}\right]$ which set the eigenvalues of $L_{1}$ as $[-7.5,-5,-4,-2]$ and those of $L_{2}$ as $[-3,-2,-1.6,-0.8]$.

Finally we have:

$$
P_{1}(\delta)=\left[\begin{array}{cc}
2 \delta^{2}+\delta+6 & 0 \\
3 \delta^{3}+2 \delta^{2}+1 & 12.5 \\
-\delta^{4}-\delta^{2}+\delta+8 & -\delta^{2}+\delta+1 \\
-2 \delta^{2}\left(\delta^{3}+\delta-1\right) & 2 \delta\left(-\delta^{2}+\delta+1\right)+37.5
\end{array}\right]
$$


and

$$
P_{2}(\delta)=\left[\begin{array}{cc}
2 \delta^{2}+\delta+2.4 & 0 \\
3 \delta^{3}+2 \delta^{2}+1 & 5 \\
-\delta^{4}-\delta^{2}+\delta+1.28 & -\delta^{2}+\delta+1 \\
-2 \delta^{2}\left(\delta^{3}+\delta-1\right) & 2 \delta\left(-\delta^{2}+\delta+1\right)+6
\end{array}\right]
$$

which yields $d_{\max }=\operatorname{deg}_{\max } P_{i}=4$.

Step 5: With a prescribed positive constant $\sigma>4 h$, we can easily design an impulsive fixed-time observer of type (12) to achieve a fixed-time estimation of $x(t)$. For the simulation, the sampling time is set as $0.0005 s$ and the basic delay $h=0.001 s$. The estimated $x(t)$ of system (2) is depicted in Figs. $1-3$ when $\sigma=0.3 s$ and in Figs. $4-6$ when $\sigma=0.5 s$.

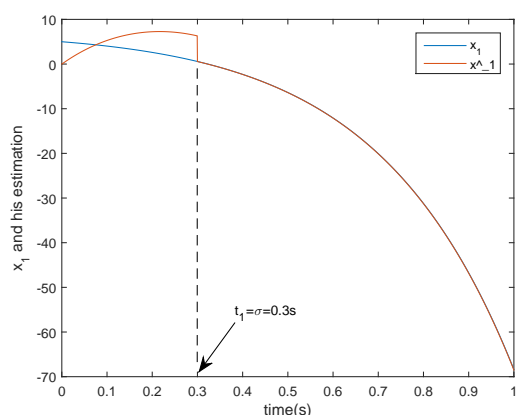

Fig. 1: $x_{1}$ and its estimation $\hat{x}_{1}$ when $\sigma=0.3 s$.

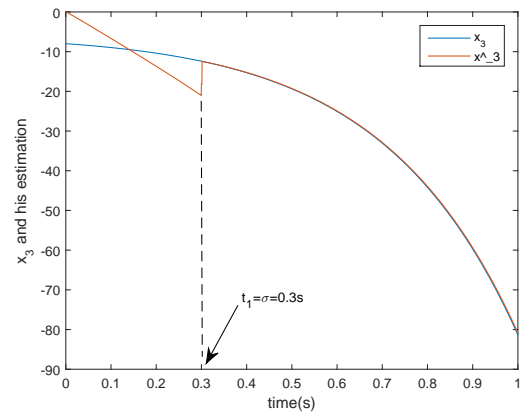

Fig. 3: $x_{3}$ and its estimation $\hat{x}_{3}$ when $\sigma=0.3 s$. Fig. $4: x_{1}$ and its estimation $\hat{x}_{1}$ when $\sigma=0.5 s$.

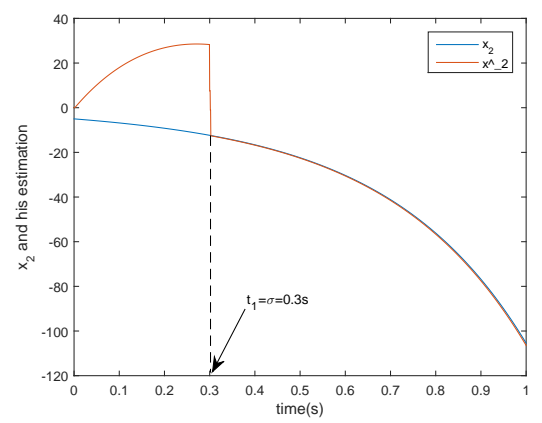

Fig. 2: $x_{2}$ and its estimation $\hat{x}_{2}$ when $\sigma=0.3 s$.

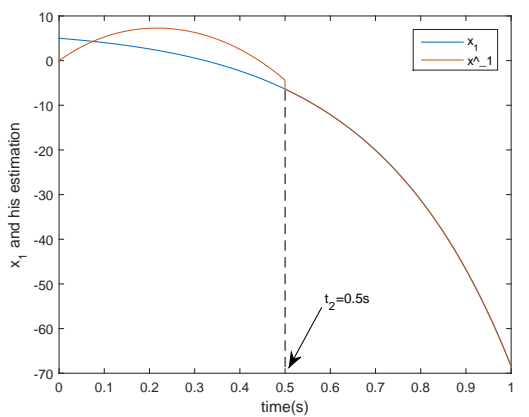



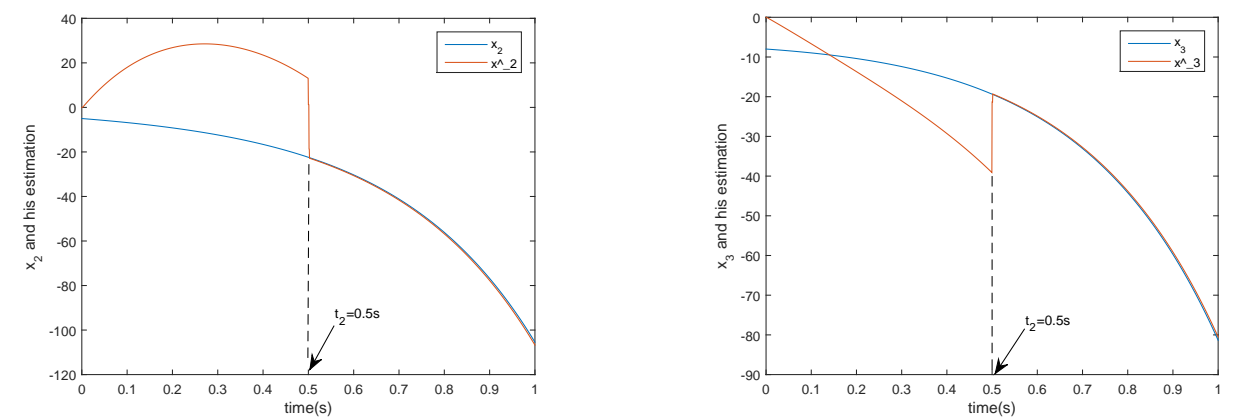

Fig. 5: $x_{2}$ and its estimation $\hat{x}_{2}$ when $\sigma=0.5 s$. Fig. 6: $x_{3}$ and its estimation $\hat{x}_{3}$ when $\sigma=0.5 s$.

From Figs. 1-3, we can see that trajectories of the proposed observer converge exactly at the prescribed time $\sigma=0.3 s$ to those of system (2). From Figs. $4-6$, the same conclusion can be made for the case $\sigma=0.5 s$.

\section{Conclusion}

In this paper, an impulsive observer with prescribed fixed-time convergence for linear systems with commensurate delay has been presented, which can involve the delays both in the state and in the output. It has been shown that the proposed observer converges in fixed time and the convergence time $\sigma$ can be chosen almost arbitrary provided that $\sigma>h \times d_{\max }$. Numerical simulations are provided for an academic example for different choices of $\sigma$ in order to highlight the feasibility of the proposed method.

\section{Reference}

Bejarano, F., Zheng, G., 2014. Observability of linear systems with commensurate delays and unknown inputs. Automatica 50 (8), 2077-2083.

Bejarano, F., Zheng, G., 2017. Observability of singular systems with commensurate time-delays and neutral terms. Automatica 85 (Supplement C), 462 - 467.

Chen, J., Xu, S., Jia, X., Zhang, B., 2017a. Novel summation inequalities and their applications to stability analysis for systems with time-varying delay. IEEE Transactions on Automatic Control 62 (5), 2470-2475.

Chen, J., Xu, S., Zhang, B., 2017b. Single/multiple integral inequalities with applications to stability analysis of time-delay systems. IEEE Transactions on Automatic Control 62 (7), 3488-3493.

Cruz-Zavala, E., Moreno, J. A., Fridman, L. M., 2011. Uniform robust exact differentiator. IEEE Transactions on Automatic Control 56 (11), 2727-2733.

Darouach, M., 2001. Linear functional observers for systems with delays in state variables. IEEE Transactions on Automatic Control 46 (3), 491-496. 
Darouach, M., Zasadzinski, M., Xu, S., 1994. Full-order observers for linear systems with unknown inputs. IEEE Transactions on Automatic and Control 39 (3), 606-609.

Emre, E., Khargonekar, P., 1982. Regulation of split linear systems over rings: Coecient-assignment and observers. IEEE Transactions on Automatic Control 27 (1), 104-113.

Engel, R., Kreisselmeier, G., 2002. A continuous-time observer which converges in finite time. IEEE Transactions on Automatic Control 47 (7), 1202-1204.

Hostetter, G., Meditch, J., 1973. Observing systems with unmeasurable inputs. IEEE Transactions on Automatic Control 18 (3), 307-308.

Hou, M., Müller, P. C., 1992. Design of observers for linear systems with unknown inputs. IEEE Transactions on Automatic Control 37 (6), 632-635.

Hou, M., Zitek, P., Patton, R. J., 2002. An observer design for linear time-delay systems. IEEE Transactions on Automatic Control 47 (1), 121-125.

Kudva, P., Viswanadham, N., Ramakrishna, A., 1980. Observers for linear systems with unknown inputs. IEEE Transactions on Automatic Control 25 (1), 113-115.

Langueh, K. A., Zheng, G., Floquet, T., 2016. Finite-time observer for linear system with time delay. In: 2016 35th Chinese Control Conference (CCC). pp. 56-60.

Luenberger, D., 1966. Observer for multivariable systems. IEEE Transactions on Automatic Control 11 (2), 190-197.

Raff, T., Allgower, F., 2007. An impulsive observer that estimates the exact state of a linear continuous-time system in predetermined finite time. Proceedings of the 15th Mediterranean Conference on Control and Automation.

Richard, J.-P., 2003. Time-delay systems: an overview of some recent advances and open problems. Automatica 39 (10), 1667-1694.

Salamon, D., 1980. Observers and duality between observation and state feedback for time delay systems. IEEE Transactions on Automatic Control 25 (6), 1187-1192.

Sename, O., 1997. Unknown input robust observer for time delay system. Proceedings of IEEE Conference on Decision and Control.

Sename, O., Briat, C., 2001. New trends in design of observers for time-delay systems. Kybernetica 37 (4), 427-458.

Seuret, A., Floquet, T., Richard, J.-P., Spurgeon, S. K., 2007. A sliding mode observer for linear systems with unknown time varying delay. In: American Control Conference. pp. $4558-4563$.

Shi, P., Zhang, Y., Agarwal, R., 2015. Stochastic finite-time state estimation for discrete time-delay neural networks with markovian jumps. Neurocomputing 151, Part 1, 168 -174 . 
Sira-Ramrez, H., Garca-Rodrguez, C., Corts-Romero, J., Luviano-Jurez, A., 2014. Algebraic Identification and Estimation Methods in Feedback Control Systems. John Wiley Sons, Ltd.

Utkin, V. I., 1992. Sliding Modes in Control Optimization. Springer-Verlag, Berlin.

Wang, S., Davison, E., Dorato, P., 1975. Observing the states of system with unmeasurable disturbances. IEEE Transaction On Automatic Control 20 (5), 716-717.

Yang, F., Wilde, R. W., 1988. Obervers for linear systems with unknown inputs. IEEE Transactions on Automatic Cotrol 33 (7), 677-681.

Zheng, G., Barbot, J.-P., Boutat, D., 2013. Identification of the delay parameter for nonlinear time-delay systems with unknown inputs. Automatica 49 (6), 1755-1760.

Zheng, G., Barbot, J.-P., Boutat, D., Floquet, T., Richard, J.-P., 2011. On observation of time-delay systems with unknown inputs. IEEE Transactions on Automatic Control 56 (8), 1973-1978.

Zheng, G., Bejarano, F., 2017. Observer design for linear singular time-delay systems. Automatica 80 (Supplement C), 1-9.

Zheng, G., Bejarano, F., Perruquetti, W., Richard, J.-P., 2015. Unknown input observer for linear time-delay systems. Automatica 61 (C), $35-43$.

Zheng, G., Boutat, D., Floquet, T., Barbot, J.-P., 2008. Secure data transmission based on multi-input multi-output delayed chaotic system. International Journal of Bifurcation and Chaos 18 (07), 2063-2072.

Zheng, G., Orlov, Y., Perruquetti, W., Richard, J. P., 2014. Finite-time-observer design for nonlinear impulsive systems with impact perturbation. International Journal of Control 87 (10), 2097-2105.

Zheng, G., Wang, H., 2016. A simple finite-time observer for linear time-delay systems. In: 2016 14th International Workshop on Variable Structure Systems (VSS). pp. 39-42. 